\title{
Sostenibilidad ecológica de la Producción Agropecuaria Tradicional de Carhuaz, Áncash, Perú
}

\author{
Ecologic sustainability of Traditional Farming of Carhuaz, Ancash, Peru \\ Juan Barreto Rodríguez ${ }^{1}$, Manuel Canto Sáenz ${ }^{2}$ y Alberto Julca Otiniano²
}

\section{RESUMEN}

El trabajo se realizó en un espacio territorial de 25,530 ha.; involucra cinco distritos (Yúngar, Anta, Marcará, Carhuaz y Ataquero) de la Cordillera Negra, en Carhuaz, con la finalidad de conocer el estado actual de los paisajes y de la sostenibilidad ecológica de la Producción Agropecuaria Tradicional (PAT) de esa zona. Para ello, se seleccionaron los indicadores más apropiados, acordes con los objetivos perseguidos; se realizó una encuesta a una muestra de 339 familias asentadas en el ámbito de estudio y, al mismo tiempo, se hizo la descripción de los paisajes del lugar. Los resultados demuestran que, en la zona de estudio se mantiene la conducción de actividades integradas; es decir cultivos, crianzas, actividad forestal y pasturas. Así mismo, se encontró la coexistencia de diferentes niveles de sostenibilidad ecológica de las unidades de producción agropecuaria tradicional.

Palabras clave: sostenibilidad ecológica; producción agropecuaria tradicional; paisaje.

\section{ABSTRACT}

The work was performed in a territorial area of 25.530 ha., Involving five districts (Yungar, Anta, Carhuaz and Ataquero) of the Black Mountain Range in Carhuaz, in order to know the current state of the landscape and the Traditional ecological sustainability of Agricultural Production (PAT) in that area. To do so, the most appropriate indicators were selected according to the objectives pursued, a survey was conducted on a sample of 339 families living in the study area and at the same time, the description of the scenery of the place was. The results show that in the study area conducting integrated activities is maintained; ie crops, raisings, forestry and pasture. Likewise, the coexistence of different levels of ecological sustainability of traditional agricultural production units found.

Keywords: ecological sustainability; traditional farming; landscape.

1 Universidad Nacional «Santiago Antúnez de Mayolo». Huaraz, Perú.

2 Universidad Nacional Agraria La Molina. Lima, Perú. 


\section{ICHIKLLACHAW}

Kay urya rukashqa Yunkar, Anta, Markaraa, Qarwash y Ataqiru, llapan kayan Yana Hirka, Qarwashchaw, alli murukuykuna kashqanta, llapan chakrakuna rikay, kaway patsatsik imanaw kashqanta, tsayman imanawshi tanqanakun unay unaypita murukuykunawan. Tsaypaq ashikashqa aalli tsaypaq willakuqkunata, tsaynawmi rukashqa 339 tapukuykunata, tsaychaw kawaqkunatahina tsayman rikapashqa kashqa llapan tsaychaw kawakuna. Tsaychawmi yarqamushqa: kay markakunachaw murukuyan hukkunatawan tsarinakushqatam: murukuy, ashmakuna waatay, hachakuna waatay, pastukuna waataytapis. Hina tsaychawmi tarikashqa kawanqantsik patsata manam llapan tsaynawllatsu tsarapaayaa.

Pushaq shimikuna: Patsantsik shumaq waatay; unaykunapita imanawpis murukuy; rikaakunapaq patsachaw kaqkuna.

\section{INTRODUCCIÓN}

Este trabajo tiene el propósito de conocer, de un lado, el nivel de sostenibilidad de los paisajes de la zona de la Cordillera Negra, en Carhuaz, zona que está destinada a la producción agropecuaria tradicional y, de otro, el nivel o estado de sostenibilidad de las tierras cultivables, las mismas que se están aprovechando desde tiempos inmemoriales, gracias al conocimiento campesino e indígena que se ha transmitido de generación en generación. La sostenibilidad de las áreas cultivables se refiere a la capacidad que tienen los agro-ecosistemas (los campos de cultivo) para mantener la producción a través del tiempo, incluso en presencia de repetidas restricciones ecológicas y presiones socio-económicas. En el caso de los paisajes, se refiere a la belleza natural que estos pueden presentar, en función de la diversidad o riqueza de flora y fauna que posean, producto del manejo que hacen los campesinos.

Cabe mencionar que a la fecha no existen trabajos de investigación de esta índole, en nuestro país; existen solamente estudios comparativos de dos cultivos o de un mismo cultivo en zonas diferentes. Por eso, la importancia del trabajo se desprende de la originalidad del tema y de la gran utilidad práctica para los diferentes actores del desarrollo agrario de la zona (campesinos, autoridades locales, autoridades sectoriales y comunidad académica en general), habida cuenta de que, al describir el estado de sostenibilidad, tanto de los paisajes como de las áreas cultivables, se puede identificar los indicadores críticos (factores que están contribuyendo a la degradación de los recursos [suelos, agua, bosques y pastizales]), a fin de tomar las decisiones orientadas a corregir o mejorar los indicadores críticos, a través del manejo, o potenciar aquellos factores que están contribuyendo positivamente. La información puede ser utilizada por los dirigentes de la comunidad o por las autoridades locales para diseñar proyectos de desarrollo agropecuario en la zona o para implementar programas de apoyo a los productores campesinos, por parte de autoridades locales y sectoriales. En tal virtud, el desarrollo del trabajo de investigación tuvo como objetivos los siguientes: 
Objetivo general: Conocer el nivel o estado de sostenibilidad, tanto de los paisajes de la Cordillera Negra, como también de las tierras cultivables de la zona.

Objetivos específicos: Establecer los indicadores más apropiados a la zona de estudio (que sean confiables y objetivamente verificables); hacer las mediciones respectivas de los indicadores, indagar las formas para conservar el agua y mejorar la fertilidad de los campos y, por último, procesar la información y evaluar la sostenibilidad ecológica.

El trabajo reviste especial importancia por las consideraciones antes mencionadas y, porque los resultados pueden ser utilizados como línea de base para futuros ensayos de investigación o para plantear nuevas hipótesis de trabajo.

\section{MATERIALES Y MÉTODOS}

El trabajo se desarrolló en el ámbito de la zona oriental de la Cordillera Negra en la provincia de Carhuaz, Áncash, abarcando el territorio de cinco distritos: Yúngar, Anta, parte de Marcará, Carhuaz y Ataquero, que involucran 25,530 ha.

Se empleó el diseño de investigación no experimental, transversal a nivel descriptivo y explicativo, que consistió en describir la realidad situacional o los valores que tienen una o más variables en el momento actual, describirlos y correlacionarlos de ser el caso (Hernández, et al., 2010). Se dice que fue transversal porque la información fue tomada en un momento único en el tiempo. Es descriptivo, porque se reporta los datos tales como son en el tiempo y el espacio y, es explicativo, porque se sustenta científicamente los hechos encontrados.

La población de estudio estuvo constituida por todas las familias que viven en el ámbito oriental de la Cordillera Negra (Callejón de Huaylas), en la provincia de Carhuaz, las mismas que están distribuidas en forma dispersa en los cinco (05) distritos antes mencionados, alcanzando un total de 2900 familias. La unidad de análisis estuvo constituida por una familia rural del ámbito de estudio y la muestra por 339 familias, que fueron encuestadas en forma aleatoria. Además se entrevistó a 2 alcaldes y 4 dirigentes de las comunidades.

Para evaluar la sostenibilidad ecológica de la PAT de Carhuaz, se seleccionó un conjunto de indicadores, los más apropiados a las condiciones del lugar, ya que los fenómenos asociados con la sostenibilidad son específicos para cada lugar evaluado (Astier et al., 2008; Nahed, 2008). La evaluación se realizó solamente en las áreas de uso agronómico (campos cultivables), es decir, no se ha tenido en cuenta las áreas de protección, ni de pastos naturales, ya que el interés se centró en saber en qué medida las áreas de producción agrícola están sufriendo procesos de degradación o se mantienen sostenidamente productivas. Para este trabajo se ha seleccionado los indicadores que evalúan el estado de sostenibilidad de los suelos y de los paisajes bajo PAT y su tendencia, los mismos que se describen a continuación. 
Tabla 1. Indicadores para la evaluación ecológica de la PAT

\begin{tabular}{|c|c|c|}
\hline & Indicadores & Caracterización \\
\hline 1. & $\begin{array}{l}\text { Prácticas de } \\
\text { conservación de } \\
\text { suelos (A) }\end{array}$ & $\begin{array}{l}\text { Se refiere a las técnicas o labores que el product } \\
\text { crementar la capacidad productiva de los suelos. } \\
\text { ellas, mayor será la sostenibilidad ecológica de la }\end{array}$ \\
\hline 2. & $\begin{array}{l}\text { Mejoramiento } \\
\text { de la calidad del } \\
\text { suelo (B) }\end{array}$ & $\begin{array}{l}\text { a fin de mejorar la salud (presencia de organismos } \\
\text { de nutrientes) del suelo. Las rotaciones y las aplica } \\
\text { orgánica, mejoran la sostenibilidad ecológica de la }\end{array}$ \\
\hline 3. & $\begin{array}{l}\text { Pendiente del } \\
\text { terreno }(C)\end{array}$ & $\begin{array}{l}\text { La pendiente del terreno tiene influencia directa en la degradación de los suelos } \\
\text { por erosión (pérdida de suelos y de su fertilidad); por eso, a mayor pendiente, } \\
\text { mayor riesgo de erosión y viceversa. Por tanto, cuanto mayor es el riesgo, menor } \\
\text { será la sostenibilidad de la PAT. }\end{array}$ \\
\hline 4 & $\begin{array}{l}\text { Cobertura del } \\
\text { terreno (D) }\end{array}$ & lá \\
\hline 5. & $\begin{array}{l}\text { Uso de } \\
\text { Agroquímicos } \\
\text { (E) }\end{array}$ & $\begin{array}{l}\text { Se refiere al nivel en que son utilizados los agroquímicos como: fertilizantes, } \\
\text { pesticidas y desinfectantes. Cuanto mayor es el uso de ellos, mayor será la con- } \\
\text { taminación y menor será la sustentabilidad ecológica. }\end{array}$ \\
\hline
\end{tabular}

\section{RESULTADOS}

\section{Sostenibilidad de los paisajes bajo PAT}

La PAT de la Cordillera Negra se desarrolla en un espacio territorial caracterizado por una gran diversidad de paisajes que corresponden a diferentes usos, incluyendo áreas silvestres o de protección. Gracias a las adecuadas prácticas de manejo tradicionales, que los campesinos aplican en la zona, los paisajes mantienen o conservan una alta biodiversidad (natural y cultivable), como es el caso de variedades nativas de papa, oca, olluco, quinua, mashua y muchas otras especies nativas y exóticas de vegetación arbórea y arbustiva (figura 1).

El espacio natural o terreno constituye el medio o recurso más importante que los campesinos disponen para producir los diversos productos alimenticios. Ellos son conscientes del valor e importancia que tiene el terreno y, por eso, lo cuidan y lo protegen incluso con su propia vida.

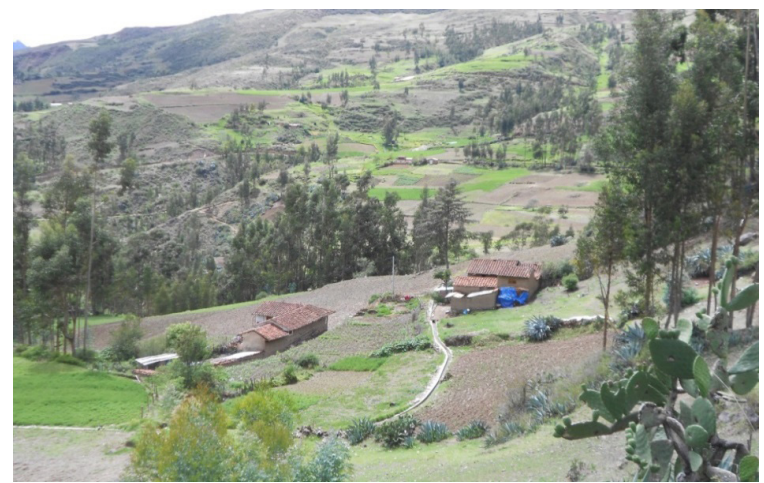

Figura 1. Vista panorámica de un paisaje rural en el ámbito de estudio (Póyor - Yúngar) 
En la figura 1 se puede apreciar un paisaje del ámbito rural estudiado, en donde se entremezclan diferentes actividades, como son: la agricultura, la ganadería y la explotación forestal, conformando un agro-ecosistema con muchos componentes que interaccionan entre sí, para configurar un bello panorama para la recreación del ser humano. Desde luego, es el hombre quien toma las decisiones del manejo de estos espacios territoriales y, de él depende el estado en que se encuentren los paisajes y los recursos (suelo, agua, bosques) que ellos disponen.

\section{Estado de la sostenibilidad ecológica del Área Cultivable}

La sostenibilidad ecológica, en este trabajo, se ha estudiado desde dos puntos de vista: desde el punto de vista del estado de los paisajes, cuya evaluación se presenta en el subtítulo anterior y, desde el punto de vista de la productividad del recurso suelo, que se expone a continuación.

Para evaluar el nivel o estado de sostenibilidad de la PAT se aplicó el método de Sarandón (2002), empleando una escala de uno (1) a cinco (5); donde: 5 es el nivel alto de sostenibilidad; 4 nivel medio; 3 nivel bajo o crítico; 2 y menos de 2 , muy bajo. Teniendo el valor de cada indicador, se determina el índice ecológico (IE) promedio de todas las chacras evaluadas, empleando la siguiente fórmula:

$$
I E=\frac{2 \mathrm{~A}+\mathrm{B}+\mathrm{C}+2 \mathrm{D}+\mathrm{E}}{7}=\frac{2(2,8)+3,5+2(2,6)+3,5}{7}=3.03
$$

En la fórmula aparecen los indicadores de la tabla 1, los cuales han sido ponderados, según la experiencia del investigador, de acuerdo a su influencia sobre la degradación de los suelos. En este caso los indicadores que tienen mayor peso son: Las prácticas de conservación (A) y la cobertura del terreno (D). El índice de sostenibilidad (IE) obtenido 3.03, nos indica que el estado promedio actual de sostenibilidad ecológica de las áreas productivas se encuentra en un límite crítico. Luego, conociendo el IE, se procede a determinar la condición o estado de cada uno de los indicadores, los mismos que se presentan en la figura 2.

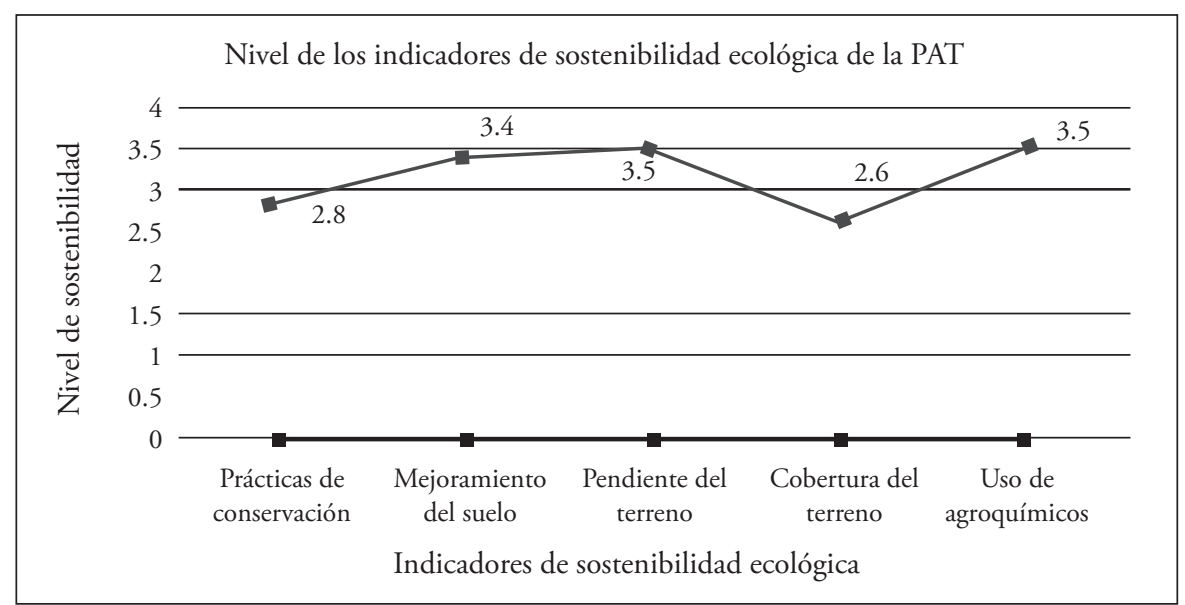

Figura 2. Estado actual de los indicadores de la sostenibilidad ecológica de las áreas cultivables 
En el gráfico se puede observar que la curva de sostenibilidad ecológica involucra los cinco indicadores antes mencionados, algunos de los cuales están por encima del nivel crítico (3) y otros por debajo. Por tanto, la curva nos permite detectar los indicadores que se encuentran en estado crítico (que están contribuyendo a la degradación del recurso suelo) y aquellos que están en mejor estado.

3. Sostenibilidad ecológica discriminada de las unidades de producción familiar (Chacras)

Se ha mencionado que con el índice de sostenibilidad ecológica (IE), se ha determinado solamente el nivel promedio actual de sostenibilidad ecológica de las áreas cultivables. Por eso, en este trabajo, también se realizó una evaluación de la sostenibilidad ecológica discriminada de las unidades de producción (chacras), cuyos resultados se exponen a continuación.

En la tabla 2 se puede observar que el 10\% de las unidades productivas estudiadas presenta un alto nivel de sostenibilidad ecológica; un 56\% de las mismas tiene una sostenibilidad ecológica media (los suelos mantienen su productividad en el tiempo); un 30,5\% se encuentra en un límite crítico, es decir, con problemas de degradación paulatina de suelos por erosión y, por último, 3,5\% de las unidades productivas está en condiciones de insostenibilidad, es decir, que los suelos están muy degradados, desprotegidos y prácticamente improductivos.

Tabla 2. Niveles de sostenibilidad ecológica de las unidades PAT de Carhuaz, Áncash, en porcentaje de chacras

\begin{tabular}{lcccc}
\hline \multirow{2}{*}{ Indicadores evaluados } & \multicolumn{4}{c}{ iveles de sostenibilidad ecológica } \\
& Alto & Medio & Bajo & Muy Bajo \\
\hline Prácticas de Conservación de suelos & 17,2 & 81,0 & 1,8 & -- \\
Mejoramiento del suelo & 0,4 & 77,1 & 21,8 & 0,7 \\
Pendiente del terreno & 10,8 & 37,7 & 50,1 & 1,4 \\
Cobertura del terreno & 14,8 & 40,4 & 37,9 & 7,0 \\
Uso de agroquímicos & 7,0 & 43,9 & 41,0 & 8,1 \\
Promedio & 10,0 & 56,0 & 30,5 & 3,5 \\
\hline
\end{tabular}

\section{Conservación de los recursos: agua y suelo}

El agua es el recurso limitante más importante para la producción en la zona y, por eso, cualquier iniciativa para aumentar la disponibilidad de agua de riego es bienvenida. Durante la aplicación de las encuestas, surgieron diferentes propuestas de los mismos campesinos, para conservar o aumentar la disponibilidad de agua de riego, ya que la zona tiene escasas fuentes. 


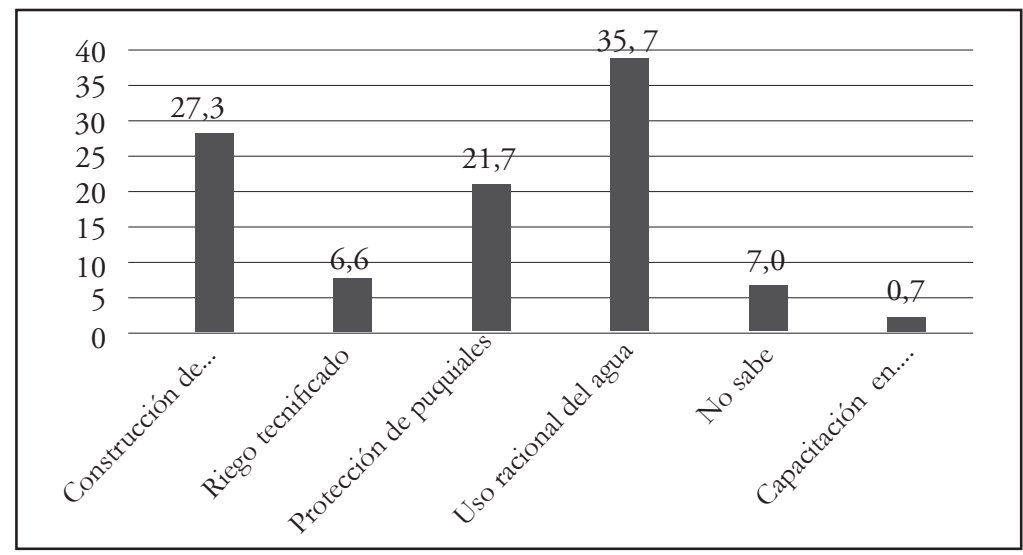

Figura 3. Propuestas espontáneas de los campesinos para conservar el agua

En la figura 3 podemos observar que el mayor porcentaje (36,7\%) de iniciativas está relacionado con el uso racional del agua; como segunda prioridad $(27,3 \%)$ está la construcción de represas y/o reservorios, como una estrategia para cosechar agua durante las lluvias y utilizarla racionalmente durante el estiaje. Un 21,7\% considera importante la protección de puquiales, también está el riego tecnificado $(6,6 \%)$ y un porcentaje mínimo $0,7 \%$ resalta la capacitación.

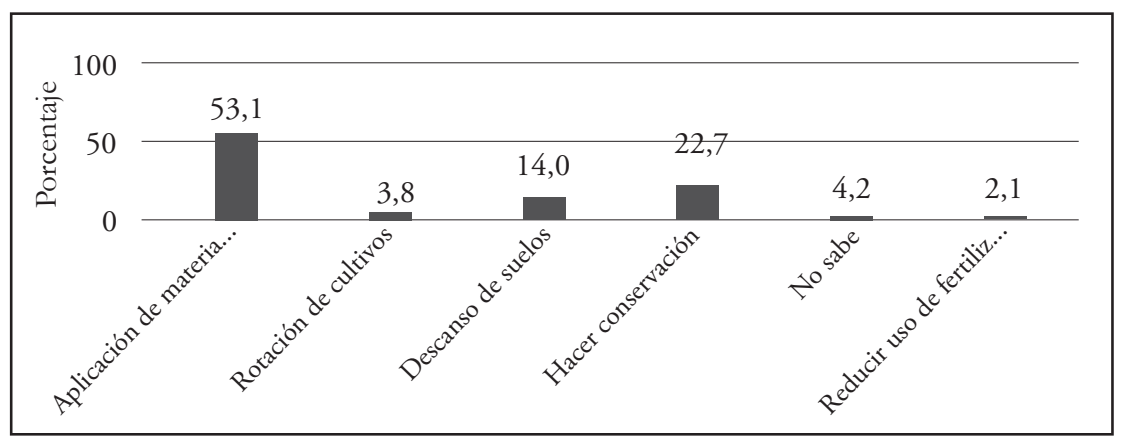

Figura 4. Como mantener la capacidad productiva del suelo

La capacidad productiva del suelo es la condición más importante de la sostenibilidad ecológica de los terrenos y, esta se puede mantener o incrementar de diferentes formas. Los campesinos mencionaron las alternativas que se muestran en la figura 4.

La mayoría de campesinos (53,1\%) considera que se puede lograr mediante la aplicación periódica de materia orgánica; un 22,7\% menciona que se logra haciendo conservación de suelos; el 14\% considera importante el descanso de los suelos; un 3,8\% recomienda la rotación de cultivos y un $2,1 \%$ sugiere reducir el uso de fertilizantes químicos.

\section{DISCUSIÓN}

La zona oriental de la Cordillera Negra, en Carhuaz, Áncash, presenta una topografía quebrada y muy heterogénea, con algunas áreas ligeramente planas, la presencia de 
mesetas a diferentes alturas y también áreas con mucha pendiente, conformando una colorida gamma de paisajes muy heterogéneos donde los campesinos conducen diferentes cultivos y crianzas y donde se puede encontrar una riquísima vegetación nativa de naturaleza herbácea y arbustiva. Este detalle concuerda con Altieri y Nicholls (2000) y Mitchell and Brown (2002), quienes mencionan que este tipo de uso, a diferentes alturas, constituye una característica típica de muchas sociedades y culturas alto-andinas.

De esos paisajes, el hombre obtiene alimentos (diversidad de productos agrícolas y pecuarios), combustibles (leña), hierbas medicinales, especies aromáticas, frutas nativas (tuna, capulí, etc.), forraje para el ganado, aire puro y agua, tanto para el consumo humano como también para los animales y para el riego de los cultivos. Los campesinos están en permanente contacto con los suelos y la naturaleza, tienen un conocimiento ancestral del manejo racional de los mismos, son conscientes de que ellos son parte de la naturaleza y que, al mismo tiempo, dependen de ella. En esos espacios territoriales, el hombre construye sus viviendas e interactúa activamente con el ecosistema, tomando las decisiones orientadas al buen manejo de los campos. Por eso, concordando con lo mencionado, Vía Campesina (2011) considera que algunos investigadores, a estas chacras las denominan fincas integradas, es decir, aquellas donde se combinan cultivos y ganadería, asociaciones y rotaciones y también la agroforestería, con lo cual se tiene altos niveles de biodiversidad funcional. Asimismo, se reporta que la diversidad es muy importante para el funcionamiento del ecosistema, pues permite un aprovechamiento óptimo de los diferentes recursos que tiene a su disposición, tales como el suelo, el agua, el espacio o la luz.

A este respecto, Crecente (2002) menciona que los paisajes rurales también pueden constituir destinos turísticos para los pobladores de las ciudades cercanas, quienes pueden recrearse con hermosos paisajes; al mismo tiempo, disfrutar del aire puro que nos brindan los agro-ecosistemas. Indica además que los agricultores, al producir alimentos, fibras y combustibles para cubrir sus necesidades o para su propio beneficio, están contribuyendo también, con su trabajo, al valor ambiental y social de las zonas rurales.

En cuanto a la sostenibilidad ecológica de las áreas cultivables, los resultados demuestran que dentro de la zona de estudio, se han encontrado coexistiendo diferentes niveles de sostenibilidad ecológica, debido tanto a factores de índole topográfica (pendiente del terreno) y tenencia de tierras (tamaño de las chacras), como también a factores sociales, como es el caso del manejo racional de la chacra realizado por los mismos campesinos, gracias al cual la chacra puede tener una mejor cobertura protectora. Esto concuerda con Altieri y Toledo (2011) y, Astier et al. (2008), quienes mencionan que los sistemas campesinos cuentan con un alto grado de diversidad de especies vegetales, presentes en sistemas de policultivos y/o modelos agroforestales, integran la obtención de diferentes productos, lo cual favorece la sostenibilidad ecológica de las áreas cultivables. Cuando un campo no es manejado racionalmente (sobrepastoreo de los campos, empleo del monocultivo, tala indiscriminada de bosques, etc.), los terrenos quedan expuestos a la degradación por erosión hídrica o eólica, provocando la disminución del rendimiento de los cultivos e incluso el deterioro del paisaje. 
Los resultados de este trabajo indican que hay tres indicadores que están contribuyendo positivamente a la sostenibilidad ecológica, es decir, a mantener o incrementar la capacidad productiva de los suelos, el primero está referido al mejoramiento de los suelos, que los campesinos suelen hacer mediante la aplicación periódica de materia orgánica y la rotación de cultivos. Ambas prácticas contribuyen a mejorar las propiedades físicas de los suelos, la retención de humedad y la actividad microbiológica de éstos; el segundo está referido a la pendiente del terreno; los campesinos saben que los suelos menos inclinados son más fértiles y, por eso, tratan de conducir sus cultivos en las áreas con menor pendiente (mesetas, terrazas naturales en los campos y áreas menos inclinadas) y un tercer indicador es el uso de un nivel bajo de agroquímicos (fertilizantes y pesticidas), solo los utilizan para determinados cultivos (comerciales) y en dosis relativamente pequeñas.

Además, según el conocimiento práctico de los campesinos, las estrategias principales orientadas a mantener o incrementar la productividad de los suelos serían, además de la aplicación periódica de materia orgánica a los suelos, la realización de prácticas de conservación de suelos (surcos en contorno, andenes, barreras vivas), el descanso de los suelos y la rotación de cultivos. Son acciones que ellos realizan durante el manejo de sus chacras y, que han constatado que provocan efectos positivos en la fertilidad de los suelos. Así mismo, se ha podido detectar que para conservar el recurso hídrico, los campesinos proponen en primer plano el uso racional del agua, lo cual expresa que en la mente de ellos está reconocida la importancia del agua de riego para sus cultivos; en segundo lugar consideran la necesidad de construir infraestructuras para almacenar agua (represas y reservorios), porque son conscientes de la temporalidad de este recurso, consideran también importante la protección de las fuentes de agua (puquiales, ojos de agua, quebradas), mediante la forestación con especies nativas, a fin de reducir la evaporación y aumentar la retención de agua durante las precipitaciones, y por último, mencionan la necesidad de avanzar hacia el riego tecnificado.

\section{CONCLUSIONES}

En la zona oriental de la Cordillera Negra, en Carhuaz, las unidades PAT presentan en forma simultánea diferentes estados de sostenibilidad ecológica, debido a la diversidad topográfica, edáfico - climática, el tamaño de las chacras, los sistemas de producción emprendidos y la destreza de los campesinos para el manejo.

El 10\% de las áreas productivas tiene una alta sostenibilidad ecológica; el 56\% tiene una sustentabilidad media (aceptable), el 30,5\% está ligeramente por debajo del límite crítico y un 3,5\% se presenta como ecológicamente no sustentable, debido principalmente a las fuertes pendientes y la degradación de los suelos por erosión.

El territorio bajo sistemas PAT presenta una amplia gama de paisajes muy heterogéneos, con una gran diversidad de flora y fauna, acorde con la altura sobre el nivel del mar, a la topografía del terreno y la disponibilidad de agua de riego.

Los campesinos son conscientes que para mantener la capacidad productiva de los suelos, es necesario aplicar periódicamente materia orgánica, realizar la rotación de cul- 
tivos y el descanso de los suelos y, para proteger o incrementar el agua de riego, mencionan la necesidad de utilizar racionalmente el agua, la construcción de reservorios y represas y la protección de puquiales con especies forestales nativas.

\section{REFERENCIAS BIBLIOGRÁFICAS}

Altieri, Miguel y Nicholls, Clara. 2000. Agroecología. Teoría y práctica para una agricultura sustentable. México D.F. Serie Textos básicos para la Formación Ambiental, PNUMA.

Altieri, Miguel \& Toledo, Víctor. 2011. La Revolución Agroecológica de América Latina: Rescatar la naturaleza, asegurar la soberanía alimentaria y empoderar al campesino. Bogotá: Consejo Latinoamericano de Ciencias Sociales (CLACSO).

Astier, Marta.; Masera, Omar y Galván-Miyoshi, Y. 2008. Evaluación de sustentabilidad: Un enfoque dinámico y multidisciplinario. Valencia. Ed. Mundiprensa, Fundación. Instituto de Agricultura Ecológica y Sustentable.

Crecente, Rafael. 2002. «Ordenación del espacio rural como instrumento de la multifuncionalidad», Jornada Autonómica de Galicia. <http.//www.libroblancoagricultura.com/ libroblanco/jautonómica/galicia/ponencias/crecente.> [Consulta: 22-10-15].

Hernández, R., Fernández, C., Baptista, P. 2010. Metodología de la Investigación. México D.F. Editorial Mc. Graw Hill. Quinta edición.

Mitchell, W.L \& Brown, P.F. 2002. Mountain peoples: adaptation and cultural persistence for a new century. <http://www.fao.org/DOCREP/004/Y3549Ee.> [Consulta: 0709-2015]

Nahed, José. 2008. «Aspectos metodológicos en la evaluación de la sostenibilidad de sistemas agrosilvopastoriles». Avances en Investigación Agropecuaria (AIA), vol. 12, No. 3. 3-19.

Sarandón, Santiago. 2002. El desarrollo y uso de indicadores para evaluar la sustentabilidad de los agroecosistemas. En Agroecología: El Camino hacia una agricultura sustentable. La Plata. Ediciones Científicas Americanas (E.C.A). 393-414.

Vía Campesina. 2011. La agricultura campesina sostenible puede alimentar al mundo. Yakarta. <http://www.viacampesina.org.> [Consulta: 20-10-15].

Fecha de recepción: 19 de noviembre 2015

Fecha de aceptación: 07 de diciembre 2015

\section{Correspondencia}

Juan Barreto Rodríguez

jubaguez@hotmail.com 